\title{
Computing turbulent flows driven by Rayleigh-Taylor and Richtmyer-Meshkov instabilities
}

\author{
Y. Zhou ${ }^{1}$, R. Rubinstein ${ }^{2}$, D. Eliason ${ }^{1}$, and W. Cabot $^{1}$ \\ ${ }^{1}$ Lawrence Livermore National Laboratory University of California Livermore, CA 94551, USA \\ 2 Computational Modeling and Simulation Branch NASA Langley Research Center Hampton, VA 23681, USA
}

Received 3 June 2002 / Accepted 5 March 2003

\begin{abstract}
We develop a two-scale turbulence model for turbulent mix induced by Rayleigh-Taylor and Richtmyer-Meshkov instabilities. In this model, the large scales are dominated by production by instability mechanisms, while small scales satisfy Kolmogorov dynamics. The model allows transfer rates in the two spectral regions to be unequal. Constraints on model coefficients that permit relaxation to a unique self-similar state in free decay and self-similar growth are derived.
\end{abstract}

Key words. instabilities - stars: supernovae: general - turbulence

\section{Introduction}

Since the classical work of Lord Rayleigh, a large body of work has been devoted to turbulent flows induced by hydrodynamic instabilities. The Rayleigh-Taylor instability (RTI) occurs when the perturbed interface between two fluids of different densities is subject to a normal pressure gradient oriented such that the pressure is higher in the lighter fluid $\rho_{2}$ than in the denser fluid $\rho_{1}$ (Rayleigh 1883; Taylor 1950). The RichtmyerMeshkov instability (RMI), occurs when a shock wave passes through a perturbed interface between two fluids (Richtmyer 1960; Meshkov 1969).

The RTI and RMI have numerous applications: they have been found to have a role in the description of the explosion of supernovae (Smarr et al. 1981; Arnett et al. 1989; Remington et al. 2000) and also have significant effects in inertial confinement fusion design (Lindl 1997). Better understanding of these instability driven turbulent flows is thus of critical importance to a variety of important scientific and engineering applications.

In this paper, we will develop a transport equation for turbulent flow induced by RTI and RMI. Transport modeling is especially relevant in astrophysical applications, where the enormous Reynolds numbers that arise (Ryutov et al. 1999) preclude direct numerical simulation. Our goal is to devise a model that not only captures the correct physics, but also permits practical engineering calculations. We follow the line of research of Hanjalić et al. (1980, hereafter HLS) and Schiestel (1987) by formulating a multiple-scale model of turbulence in which the transport equations for turbulence kinetic energy and

Send offprint requests to: R. Rubinstein,

e-mail: r.rubinstein@larc.nasa.gov dissipation rate of a standard two-equation turbulence model are each replaced by transport equations for the kinetic energy and dissipation rate pertaining to a definite range of scales of motion.

Multiple-scale modeling attempts to treat the response of turbulence to changes in the large-scale motion more realistically than the single-scale two-equation model. Whereas the two-equation model assumes that the inertial range can adjust instantaneously to changes at the large scales, multiple-scale modeling allows time delays in this response and thereby permits a more realistic desciption of the time-dependent behavior of turbulence.

Multiple-scale effects are expected to be important in turbulent flows with strong non-equilibrium effects and in flows with distinct dynamics at different scales of motion. RTI- and RMIdriven turbulence exhibit both properties since (Zhou 2001) the large scales are dominated by production by external agencies, whereas the small scales revert to high Reynolds number Kolmogorov dynamics; because of these different dynamic mechanisms, equilibrium between these scales should not be assumed.

Although multiple-scale models are important when significant non-equilibrium effects exist in the energy dynamics, when such effects are absent, the multiple-scale model should reduce to a single-scale model. We apply this principle in two different ways: first, we investigate conditions under which the two-scale model for decaying turbulence relaxes to a unique self-similar solution. Second, we investigate the uniqueness of the long-time self-similar growth of RTI-driven turbulence. This analysis is also pertinent to the realizability of the model. In both cases, the analysis leads to a simple inequality among the model constants. 


\section{Derivation of multiple-scale models}

The starting point is the isotropic part of the spectral evolution equation for homogeneous turbulence (Besnard et al. 1996)

$\dot{E}(k)=T(k)+\Pi(k)-D(k)$

where $k$ is the wavenumber, $E(k)$ is the energy spectrum, $T(k)$ is energy transfer spectrum, $\Pi(k)$ is the production spectrum, and $D(k)$ is the dissipation spectrum. The dissipation spectrum is defined by

$D(k)=2 v k^{2} E(k)$,

where $v$ is the kinematic viscosity. Closures must be provided for $T(k)$ and $\Pi(k)$.

The integrated quantities are

$$
\begin{aligned}
& \int_{0}^{\infty} E(k) \mathrm{d} k=K \\
& \int_{0}^{\infty} T(k) \mathrm{d} k=0 \\
& \int_{0}^{\infty} \Pi(k) \mathrm{d} k=P \\
& \int_{0}^{\infty} D(k) \mathrm{d} k=\epsilon
\end{aligned}
$$

where $K$ is the kinetic energy of turbulence, $P$ is the total production, and $\epsilon$ is the dissipation rate. The second relation in Eq. (3) expresses the conservation of energy by nonlinear interaction. The energy equation follows from integrating Eq. (1) over all wavenumbers. Using Eqs. (3),

$\dot{K}=P-\epsilon$.

This result is independent of the analytical form of $E(k)$ and $\Pi(k)$, and of the closure used to define $T(k)$.

In a standard two-equation model, the energy equation, Eq. (4), would be supplemented by an evolution equation for $\epsilon$. Because only one length or time scale can be formed from the two turbulence descriptors $K$ and $\epsilon$, the two-equation model can be considered to be a single-scale model.

One possible approach to multiple-scale modeling is to consider a piecewise-Kolmogorov spectrum

$E_{i}(k)=C_{K} \epsilon_{i}^{2 / 3} k^{-5 / 3}$ for $k_{i-1} \leq k \leq k_{i}$ with $1 \leq i \leq n$

and to set

$T(k)=-\sum_{1 \leq i \leq n} \epsilon_{i}\left[\delta\left(k-k_{i-1}\right)-\delta\left(k-k_{i}\right)\right]$.

Substituting Eqs. (5) and (6) in the spectral evolution equation, Eq. (1), would lead to a shell model of turbulence, in which energy is transferred from each discrete region of scales to the adjacent region of smaller scales (see for example Bell \& Nelkin 1978). This picture oversimplifies the actual energy transfer process, which is mediated by triad interactions (Zhou 1993a, 1993b, 1996) and which permits (Kraichnan 1959, 1971) both forward and backward energy transfer and significant transfer between non-adjacent regions.
Integration of Eq. (2) over the regions $k_{i-1} \leq k<k_{i}$, gives piecewise energy equations of the form

$\dot{K}_{i}=P_{i}+\epsilon_{i-1}-\epsilon_{i}-D_{i}$

where $D_{i}$ can be computed from Eq. (2), $P_{i}$ can be closed given a model of the production process, and we set $\epsilon_{0}=0$. Unlike Eq. (4), Eq. (7) is not an identity; it depends crucially on the assumption that energy is transferred between adjacent spectral regions only. However, the sum of Eq. (7) over all regions $i$ correctly recovers the energy balance Eq. (4).

As in the two-equation model, the partial energy equations, Eq. (7), must be supplemented by partial dissipation equations. The derivation of these equations is less straightforward. Among many proposals (for example, Cadiou 1999; Laporta 1995) we follow Schiestel's (1987) intuitive approach. Equation (5) implies

$K_{i}=\frac{3}{2} C_{K} \epsilon_{i}^{2 / 3}\left(k_{i-1}^{-2 / 3}-k_{i}^{-2 / 3}\right)$

from which differentiation in time gives

$$
\begin{aligned}
\dot{K}_{i}= & C_{K} \epsilon_{i}^{-1 / 3}\left(k_{i-1}^{-2 / 3}-k_{i}^{-2 / 3}\right) \dot{\epsilon}_{i} \\
& -C_{K} \epsilon_{i}^{2 / 3} k_{i-1}^{-5 / 3} \dot{k}_{i-1}+C_{K} \epsilon_{i}^{2 / 3} k_{i}^{-5 / 3} \dot{k}_{i} .
\end{aligned}
$$

Schiestel (1987, Eq. (24)) proposes that $\dot{k}_{i}$ be determined as the ratio of the flux into the region $k_{i} \leq k \leq k_{i+1}$ divided by the local energy density at $k_{i}$. In the present notation this leads to

$\dot{k}_{i}=\eta \frac{\epsilon_{i}}{E_{i+1}\left(k_{i}\right)}=\eta \frac{\epsilon_{i}}{C_{K} \epsilon_{i+1}^{2 / 3}} k_{i}^{5 / 3}$,

where $\eta$ is a constant and the choice of $E_{i+1}$, the energy density for scales greater than $k_{i}$, seems to be the most natural.

Substituting Eq. (10) into Eq. (9) leads to

$\dot{K}_{i}=\frac{2}{3} \frac{K_{i}}{\epsilon_{i}} \dot{\epsilon}_{i}-\eta \epsilon_{i-1}+\eta \epsilon_{i}^{2 / 3} \frac{\epsilon_{i}}{\epsilon_{i+1}^{2 / 3}}$

which can be rearranged as the piecewise $\epsilon$ equation

$$
\begin{aligned}
\dot{\epsilon}_{i} & =\frac{3}{2} \frac{\epsilon_{i}}{K_{i}}\left(P_{i}-\epsilon_{i}\right)+\frac{3}{2} \eta \frac{\epsilon_{i-1} \epsilon_{i}}{K_{i}}-\frac{3}{2} \eta \frac{\epsilon_{i}^{2 / 3}}{\epsilon_{i+1}^{2 / 3}} \frac{\epsilon_{i}^{2}}{K_{i}} \\
& =\frac{3}{2} \frac{\epsilon_{i}}{K_{i}} P_{i}+\frac{3}{2} \eta \frac{\epsilon_{i-1} \epsilon_{i}}{K_{i}}-\frac{3}{2} \frac{\epsilon_{i}^{2}}{K_{i}}\left[1+\eta \frac{\epsilon_{i}^{2 / 3}}{\epsilon_{i+1}^{2 / 3}}\right] .
\end{aligned}
$$

This result differs slightly from Eq. (25) of Schiestel (1987) because of the final term which modifies the sink term. $P_{i}$ can be neglected except in the regions with small $i$ and $D_{i}$ can be neglected except in the regions with large $i$; in the intermediate regions, the energy content is determined by nonlinear energy transfer alone. It is therefore a reasonable approximation to set $P_{i}=0$ for $i \geq 2$ and $D_{i}=0$ for $i<n$. Then the production of $\epsilon_{i}$ comes primarily from the term proportional to $\epsilon_{i-1}$, the flux into the region $k \geq k_{i}$ from the region $k<k_{i}$.

If there are only two spectral regions, a "production" region with partial energy $K_{\mathrm{p}}$ and partial dissipation $\epsilon_{\mathrm{p}}$ and a "transfer", or small-scale region with partial energy $K_{\mathrm{t}}$ and partial 
dissipation $\epsilon_{\mathrm{t}}$ equal to the viscous dissipation, then introducing these approximations in Eqs. (7) and (12) gives the fourequation model

$$
\begin{aligned}
\dot{K}_{\mathrm{p}} & =P-\epsilon_{\mathrm{p}} \\
\dot{K}_{\mathrm{t}} & =\epsilon_{\mathrm{p}}-\epsilon_{\mathrm{t}} \\
\dot{\epsilon}_{\mathrm{p}} & =C_{\mathrm{p} 1} \frac{\epsilon_{\mathrm{p}}}{K_{\mathrm{p}}} P-C_{\mathrm{p} 2} \frac{\epsilon_{\mathrm{p}}^{2}}{K_{\mathrm{p}}} \\
\dot{\epsilon}_{\mathrm{t}} & =C_{\mathrm{t} 1} \frac{\epsilon_{\mathrm{p}} \epsilon_{\mathrm{t}}}{K_{\mathrm{t}}}-C_{\mathrm{t} 2} \frac{\epsilon_{\mathrm{t}}^{2}}{K_{\mathrm{t}}}
\end{aligned}
$$

where

$$
\begin{aligned}
C_{\mathrm{p} 1} & =3 / 2 \\
C_{\mathrm{p} 2} & =\frac{3}{2}\left[1+\eta \frac{\epsilon_{\mathrm{p}}^{2 / 3}}{\epsilon_{\mathrm{t}}^{2 / 3}}\right] \\
C_{\mathrm{t} 1} & =(3 / 2) \eta \\
C_{\mathrm{t} 2} & =3 / 2 .
\end{aligned}
$$

The general model Eq. (13) was presented on basically heuristic grounds by HLS. The most important feature of this model is that production in the region $t$ is due to energy transfer from the $p$ region, rather than by the direct effect of production agencies. Consequently, the two spectral regions need not be in equilibrium. Two extreme non-equilibrium states are possible initially: the first dominated entirely by the production mechanism so that $K_{\mathrm{t}}$ and $\epsilon_{\mathrm{t}}$ both vanish initially, and the second in which the linear production mechanism is not yet effective so that $K_{\mathrm{p}}$ and $\epsilon_{\mathrm{p}}$ both vanish initially; in either case, the model would allow the other spectral region to be generated during evolution.

The formal structure of the $\mathrm{p}$ equation closely resembles the usual two-equation model. In multiple-scale models, the effect of the $t$ region on the $\mathrm{p}$ region is generally indirect: it occurs as dependence of the model constant $C_{\mathrm{p} 2}$ on $K_{\mathrm{t}}$ and/or $\epsilon_{\mathrm{t}}$. Equation (14) provides one example of this dependence, which tends to promote the equilibration of $\epsilon_{\mathrm{p}}$ and $\epsilon_{\mathrm{t}}$. We stress, however, that this derivation has been presented only to exhibit some of the ideas behind multiple-scale models, not to advocate Eq. (14) as a model; indeed, because of its very schematic picture of energy transfer, this model does not satisfy some constraints which we derive later.

\section{Transport model for turbulent mix induced by RTI and RMI}

Our goal is to apply the two-scale model to RMI- and RTIdriven turbulence. As noted in the Introduction, we believe that this application is natural, and perhaps even essential, because in RTI and RMI there actually is a fundamental dynamic difference between the large and the small scales.

The basic premise of the theory of RTI- and RMI-driven turbulence and related systems (Chandrasekhar 1961) is that turbulence is initiated by linear instabilities: there are solutions of the linearized equations of motion of the form

$u_{i}(\boldsymbol{x}, t)=A_{i} \exp [i \boldsymbol{k} \cdot \boldsymbol{x}+t / \tau(\boldsymbol{k})]$ where $u_{i}$ denotes all relevant fluctuating fields. When the linear time scale $\tau(\boldsymbol{k})$ has a positive real part, we have the case of absolute instability, which is the case of interest. Note that the exponential solution, Eq. (15), is considerably simpler than comparable result for shear turbulence, in which the instability originates in the properties of the mean velocity profile, and we must deal with complex models like the Rayleigh and Orr-Sommerfeld equation.

The classical (Kolmogorov 1941) phenomenology has been extended to treat external time scales and the nonlinear time scale on an equal footing (Matthaeus 1989; Zhou, 1995). For the RTI-driven flow, the external time scale is that associated with the linear instabilities,

$\tau_{\mathrm{RT}}=(k g A)^{-1 / 2}$

where $g$ is the acceleration and the Atwood number $A$ is given by

$A=\frac{\rho_{1}-\rho_{2}}{\rho_{1}+\rho_{2}}$,

where $\rho_{1}$ and $\rho_{2}$ are the densities of the two fluids. The external time scale associated with the RMI is

$\tau_{\mathrm{RM}}=(k A V)^{-1}$

where $V$ is the velocity jump.

Assuming that RTI-driven flow can be idealized as hydrodynamic turbulence with the special imposed linear time scale Eq. (16), we find the steady-state energy spectrum (Zhou 2001)

$E(k, z)=C_{\mathrm{RT}}(g A)^{1 / 4} \epsilon(z)^{1 / 2} k^{-7 / 4}$,

where $z$ denotes the acceleration direction.

For turbulent flow induced by RMI, we find that

$E(k, z)=C_{\mathrm{RM}}[A V \epsilon(z)]^{1 / 2} k^{-3 / 2}$,

where now $z$ is the direction of the shock. Both $C_{\mathrm{RT}}$ and $C_{\mathrm{RM}}$ are constants.

The analysis leading to the spectrum Eq. (18) applies to an idealized steady state condition established through the equilibration of production by the instability mechanisms and turbulent nonlinear energy transfer. Transient states can be expected to have a more complex structure. Thus, in developing RTI-driven flow, small scale instabilities will experience the most rapid linear growth in view of Eq. (16). But for large $k$, the nonlinear time scale $\sim k^{-2 / 3}$ is smaller than the linear time scale $\sim k^{-1 / 2}$; accordingly, the small scales will quickly saturate and reach a state of ordinary Kolmogorov turbulence. These simple heuristic considerations are consistent with the more precise weakly nonlinear analysis of Haan (1991). The situation is entirely different for large scales, for which the linear time scale is smaller; these scales can be expected to remain in a state described by Eq. (18), even if their initial linear growth is relatively slow.

It follows that in developing RTI-driven flow, we must expect the coexistence of ordinary Kolmogorov turbulence at the small scales with instability-driven turbulence described by Eq. (18) at the large scales. In order to describe this mixed state, as in the last section, turbulent fluctuations are now partitioned 
into the two regions $\mathrm{p}$ (production) and $\mathrm{t}$ (transfer). To close the equation for $K_{\mathrm{p}}$ in Eq. (13), we require a model for the production term. Following the classical work (Ledoux et al. 1961), the RTI production is given by

$P_{\mathrm{RT}}(z)=\int \mathrm{d} k(g A)^{1 / 2} k^{1 / 2} E(k)_{\mathrm{RT}}$.

It must be admitted that assuming the spectrum in Eq. (18) excludes from consideration developing states in which the large scale spectrum has not yet evolved to this form. In particular, it would be inappropriate to close the production term by Eq. (20) in an analysis of the onset of RTI-driven turbulence from a state of rest. Although this fact certainly limits the applicability of the model, it should be stressed that evolution in which the energy spectrum takes a completely nonuniversal form could not be rigorously described by any model containing a small number of turbulence descriptors.

In order to illustrate the two-scale model in as simple as possible a setting, we will consider the evolution of all turbulence quantities integrated over the $z$ direction. Although integration entails loss of information about the evolution, it is an energy conserving approximation. It can be compared to the use of quantities like turbulence kinetic energy in turbulence models, since kinetic energy is just the integral over wavenumber of the energy spectrum. The effect is to make the analysis spatially homogeneous, thereby avoiding the subtle questions of turbulent diffusion for RTI- and RMI-driven turbulence.

We define the $z$-averaged production as

$P_{\mathrm{RT}}=\int_{-\infty}^{\infty} \mathrm{d} z P_{\mathrm{RT}}(z)^{2} / \int_{-\infty}^{\infty} \mathrm{d} z P_{\mathrm{RT}}(z)$

Since the turbulence production vanishes rapidly for large $z$, the integrals in Eq. (21) are finite. Substituting the energy spectrum Eq. (18) we obtain

$P_{\mathrm{RT}}=\left(\frac{16}{3}\right)^{1 / 3} C_{\mathrm{RT}}^{1 / 3}(g A)^{2 / 3} \epsilon_{\mathrm{p}}^{1 / 3} K_{\mathrm{p}}^{1 / 3}$

where the $z$-averaged dissipation rate $\epsilon_{\mathrm{p}}$ is defined so that

$\epsilon_{\mathrm{p}}{ }^{1 / 2}=\int_{-\infty}^{\infty} \mathrm{d} z \epsilon(z) / \int_{-\infty}^{\infty} \mathrm{d} z \epsilon(z)^{1 / 2}$.

The impulsive RMI production spectrum has the form

$P_{\mathrm{RM}}=\int \mathrm{d} k(A V) k E(k)_{\mathrm{RM}}$.

This source term is effective only when the shock arrives and will vanish after the passage of the shock.

Using the production term Eq. (22) in the $K_{\mathrm{p}}$ and $\epsilon_{\mathrm{p}}$ equations, we obtain the two-scale model for RTI-driven turbulence

$$
\begin{aligned}
\dot{K}_{\mathrm{p}} & =P_{\mathrm{RT}}-\epsilon_{\mathrm{p}} \\
\dot{K}_{\mathrm{t}} & =\epsilon_{\mathrm{p}}-\epsilon_{\mathrm{t}} \\
\dot{\epsilon}_{\mathrm{p}} & =C_{\mathrm{p} 1} \frac{\epsilon_{\mathrm{p}}}{K_{\mathrm{p}}} P_{\mathrm{RT}}-C_{\mathrm{p} 2} \frac{\epsilon_{\mathrm{p}}^{2}}{K_{\mathrm{p}}} \\
\dot{\epsilon}_{\mathrm{t}} & =C_{\mathrm{t} 1} \frac{\epsilon_{\mathrm{p}} \epsilon_{\mathrm{t}}}{K_{\mathrm{t}}}-C_{\mathrm{t} 2} \frac{\epsilon_{\mathrm{t}}^{2}}{K_{\mathrm{t}}} .
\end{aligned}
$$

Again, as with Eq. (13), a transient or initial state in which $K_{\mathrm{t}}$ and $\epsilon_{\mathrm{t}}$ both vanish is possible; indeed, this might be a reasonable model for RTI growth from an initially quiescent state. The model equations (Eqs. (25)) will then generate the second spectral region as the flow develops.

For RMI, it is noted in Zhou (2001) that after the passage of a planar shock, the turbulent flow generated by RMI is essentially that of incompressible freely decaying turbulence. Therefore, for RMI-driven turbulence we simply have the equations for free decay,

$$
\begin{aligned}
\dot{K}_{\mathrm{p}} & =-\epsilon_{\mathrm{p}} \\
\dot{K}_{\mathrm{t}} & =\epsilon_{\mathrm{p}}-\epsilon_{\mathrm{t}} \\
\dot{\epsilon}_{\mathrm{p}} & =-C_{\mathrm{p} 2} \frac{\epsilon_{\mathrm{p}}^{2}}{K_{\mathrm{p}}} \\
\dot{\epsilon}_{\mathrm{t}} & =C_{\mathrm{t} 1} \frac{\epsilon_{\mathrm{p}} \epsilon_{\mathrm{t}}}{K_{\mathrm{t}}}-C_{\mathrm{t} 2} \frac{\epsilon_{\mathrm{t}}^{2}}{K_{\mathrm{t}}}
\end{aligned}
$$

where the initial conditions are determined from the instantaneous spectrum Eq. (19) Since the turbulence quantities $K_{\mathrm{p}}, K_{\mathrm{t}}$, $\epsilon_{\mathrm{p}}$, and $\epsilon_{\mathrm{t}}$ in Eq. (25) are all averages over the height variable $z$, the diffusion terms which would appear in the transport equations for $K_{\mathrm{t}}(z), \epsilon_{\mathrm{t}}(z), K_{\mathrm{p}}(z)$, and $\epsilon_{\mathrm{p}}(z)$ integrate to zero.

\section{Relaxation to self-similar solutions}

The richer dynamics possible for a multiple-scale model is potentially very useful, but it also opens the possibility of spurious solutions. Thus, if a turbulent flow relaxes to a self-similar evolution, any multiple-scale model must relax accordingly to a unique similarity solution. Such relaxation is almost automatically a dimensional necessity for single-scale models, and indeed two-equation models are calibrated based on a suite of self-similar flows.

But relaxation to a unique similarity solution is never a foregone conclusion for multiple-scale models: the reason is the appearance of dimensionless parameters like $K_{\mathrm{p}} / K_{\mathrm{t}}$ and $\epsilon_{\mathrm{p}} / \epsilon_{\mathrm{t}}$. Consequently, multiple-scale must be constrained to prevent the existence of multiple solutions. We will investigate this problem in the cases of free decay, which pertains to RMIdriven turbulence, and of self-similar growth of RTI-driven turbulence.

Consider decaying turbulence described by the two-scale model Eq. (26). Look for a solution

$$
\begin{aligned}
K_{\mathrm{p}} & =a_{\mathrm{p}} t^{\alpha} \\
K_{\mathrm{t}} & =a_{\mathrm{t}} t^{\alpha} \\
\epsilon_{\mathrm{p}} & =e_{\mathrm{p}} t^{\alpha-1} \\
\epsilon_{\mathrm{t}} & =e_{\mathrm{t}} t^{\alpha-1} .
\end{aligned}
$$

Substituting into Eq. (26) leads to a system of homogeneous equations in the constants $a_{\mathrm{p}}, a_{\mathrm{t}}, e_{\mathrm{p}}, e_{\mathrm{t}}$, which has a nontrivial solution provided

$\left|\begin{array}{cccc}\alpha & 0 & 1 & 0 \\ 0 & \alpha & -1 & 1 \\ \alpha-1 & 0 & C_{\mathrm{p} 2} & 0 \\ 0 & \alpha-1 & -C_{\mathrm{t} 1} & C_{\mathrm{t} 2}\end{array}\right|=\left(\alpha C_{\mathrm{t} 2}-\alpha+1\right)\left(\alpha C_{\mathrm{p} 2}-\alpha+1\right)=0 .(28)$ 


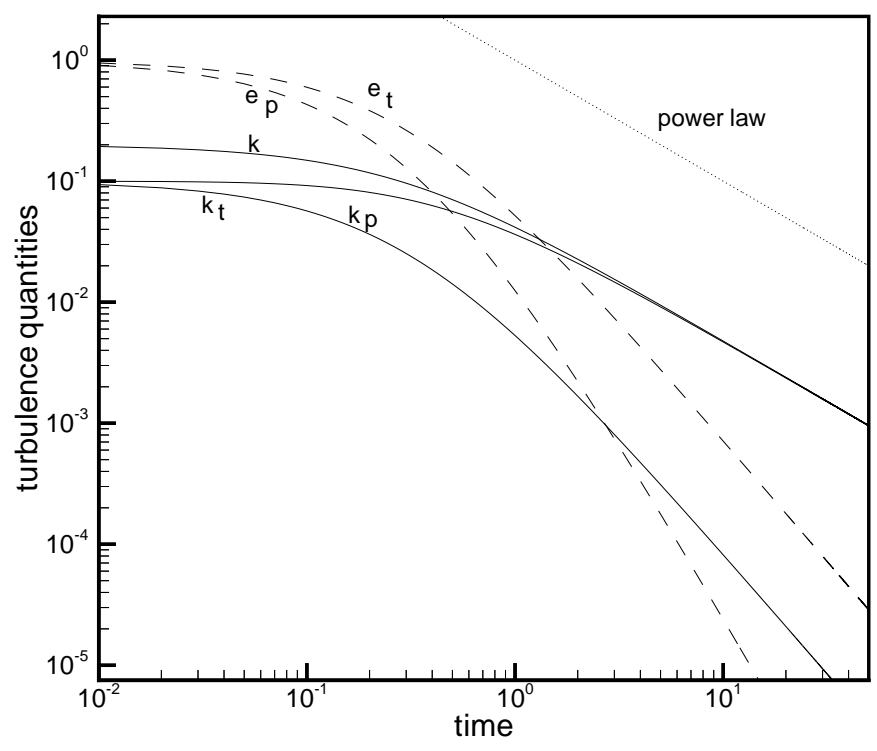

Fig. 1. The result of numerically integrating the decay when Eq. (30) is satisfied, using $C_{\mathrm{p} 2}=1.5, C_{\mathrm{t} 1}=1.2, C_{\mathrm{t} 2}=2.0$, and initial conditions $K_{\mathrm{p}}(0)=K_{\mathrm{t}}(0)=0.1, \epsilon_{\mathrm{p}}(0)=\epsilon_{\mathrm{t}}(0)=1.0$.

The solutions are $\alpha=-1 /\left(C_{\mathrm{t} 2}-1\right)$ and $\alpha=-1 /\left(C_{\mathrm{p} 2}-1\right)$ and the corresponding amplitude ratios are easily found to be

$$
\begin{aligned}
& a_{\mathrm{p}}: a_{\mathrm{t}}=\quad 0: 1 \quad \text { if } \alpha=-1 /\left(C_{\mathrm{t} 2}-1\right) \\
& a_{\mathrm{p}}: a_{\mathrm{t}}=C_{\mathrm{p} 2}-C_{\mathrm{t} 2}: C_{\mathrm{t} 2}-C_{\mathrm{t} 1} \text { if } \alpha=-1 /\left(C_{\mathrm{p} 2}-1\right) \text {. }
\end{aligned}
$$

The first solution in Eq. (29) corresponds to the reduction of the two-scale model to a single-scale model since $K_{\mathrm{p}}=\epsilon_{0}=0$. However, there is also a second solution, which as pointed out by a referee, is a consequence of the existence of an integral $K_{\mathrm{p}} \epsilon_{\mathrm{p}}^{-C_{\mathrm{p} 2}}$ of the Eqs. (26). To avoid the existence of a second power law in decaying turbulence, this solution must be nonrealizable or unstable; its non-realizability is assured if one of the amplitudes $a_{\mathrm{p}}$ or $a_{\mathrm{t}}$ must be negative. Non-realizability of the second solution is guaranteed if either

$C_{\mathrm{p} 2}<C_{\mathrm{t} 2}$ and $C_{\mathrm{t} 1}<C_{\mathrm{t} 2}$,

or

$C_{\mathrm{p} 2}>C_{\mathrm{t} 2}$ and $C_{\mathrm{t} 1}>C_{\mathrm{t} 2}$.

Assuming, following Hanjalić et al. (1980) that $C_{\mathrm{t} 2} \geq$ $C_{\mathrm{t} 1}$, relaxation to a unique self-similar decaying state requires $C_{\mathrm{p} 2}<C_{\mathrm{t} 2}$.

These inequalities on the model constants insure two important properties: that a unique power-law solution for decay exists, and that this unique solution corresponds to single-scale behavior. The first inequality in Eq. (30), $C_{\mathrm{p} 2}<C_{\mathrm{t} 2}$, has a more general significance: it states that in a model with only nearest-neighbor energy transfer, nonlinearity drives the transfer rates $\epsilon_{i}$ to zero more quickly as $i$ increases.

To illustrate the role of the constraint expressed by Eq. (30), the decay equations were numerically integrated when Eq. (30) is satisfied. The results are shown in Fig. 1. The model constants were arbitrarily chosen as $C_{\mathrm{p} 2}=1.5, C_{\mathrm{t} 1}=1.2, C_{\mathrm{t} 2}=$ 2.0 and the initial conditions were $K_{\mathrm{p}}(0)=K_{\mathrm{t}}(0)=0.1$,

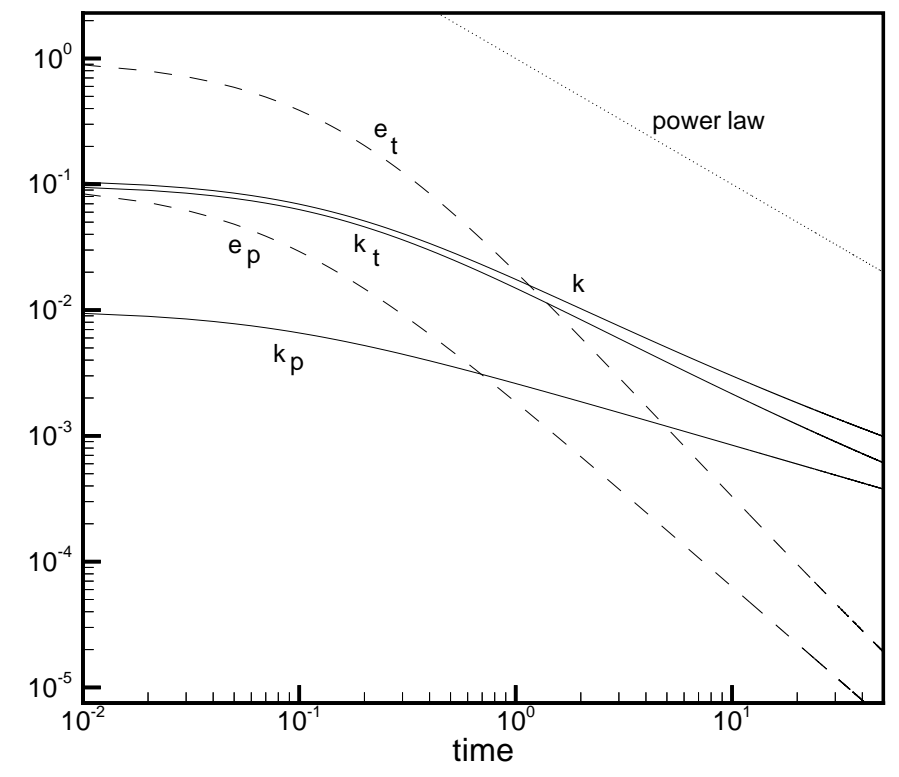

Fig. 2. As for Fig. 1, but using $C_{\mathrm{p} 2}=3.0$ and $K_{\mathrm{p}}(0)=0, \epsilon_{\mathrm{p}}(0)=0$.

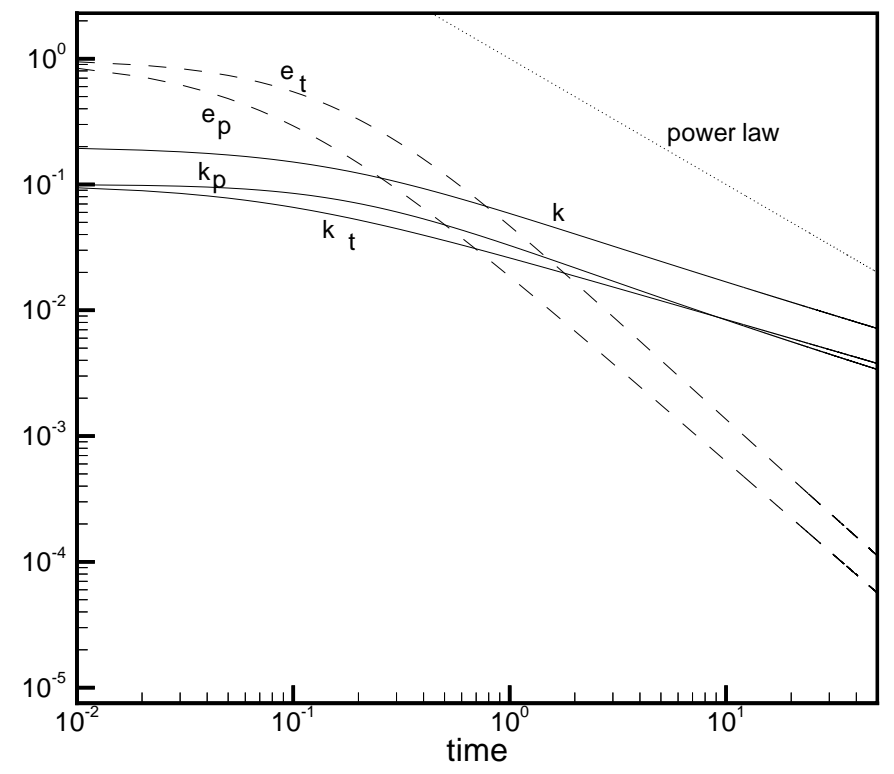

Fig. 3. As for Fig. 2, but with initial conditions $K_{\mathrm{p}}(0)=K_{\mathrm{t}}(0)=$ $0.1, \epsilon_{\mathrm{p}}(0)=\epsilon_{\mathrm{t}}(0)=1.0$.

$\epsilon_{\mathrm{p}}(0)=\epsilon_{\mathrm{t}}(0)=1.0$. Figure 1 shows that after an initial transient, the energy $K_{\mathrm{p}}$ approaches zero much faster than $K_{\mathrm{t}}$, indicating that at long times the multiple-scale model reduces to a single-scale model. The dotted line shows the power law decay $K \sim t^{-1}$ expected in this case.

To demonstrate that Eq. (30) is needed because the second solution in Eq. (29) can be stable, the decay equations were integrated for a case which does not satisfy the constraint, $C_{\mathrm{p} 2}=3.0, C_{\mathrm{t} 1}=1.2, C_{\mathrm{t} 2}=2.0$. The choice of constants is purely illustrative; in fact, in the model Eq. (13), Eq. (14) states that $C_{\mathrm{p} 2}$ is not constant: an example with variable $C_{\mathrm{p} 2}$ is considered below. The results are shown in Figs. 2 and 3. In Fig. 2, the initial conditions are $K_{\mathrm{p}}(0)=0, K_{\mathrm{t}}(0)=$ $0.1, \epsilon_{\mathrm{p}}(0)=0, \epsilon_{\mathrm{t}}(0)=1.0$ while in Fig. 3 the initial conditions 
are $K_{\mathrm{p}}(0)=K_{\mathrm{t}}(0)=0.1, \epsilon_{\mathrm{p}}(0)=\epsilon_{\mathrm{t}}(0)=1.0$. Clearly, the power law decay is different in each case; the dotted line again corresponds to the power law $K \sim t^{-1}$. Thus, if the conditions expressed by Eq. (30) are not satisfied, two distinct time-scaling laws can exist for decaying turbulence.

The original proposal of HLS was

$$
\begin{aligned}
C_{\mathrm{p} 2} & =1.8-0.3 \frac{K_{\mathrm{p}} / K_{\mathrm{t}}-1}{K_{\mathrm{p}} / K_{\mathrm{t}}+1} \\
C_{\mathrm{t} 1} & =1.08 \frac{\epsilon_{\mathrm{p}}}{\epsilon_{\mathrm{t}}} \\
C_{\mathrm{t} 2} & =1.15 .
\end{aligned}
$$

We note that like the comparable dependence in Eq. (12), the variable $C_{\mathrm{p} 2}$ tends to promote the equilibrium $\epsilon_{\mathrm{p}}=\epsilon_{\mathrm{t}}$; however, Eq. (32) proposes an energy-based modification of $C_{\mathrm{p} 2}$ instead of a dissipation rate-based modification.

HLS found that during decay,

$K_{\mathrm{p}}: K_{\mathrm{t}}=\left(C_{\mathrm{p} 2}-C_{\mathrm{t} 2}\right):\left(C_{\mathrm{t} 2}-C_{\mathrm{t} 1}\right)$

where

$K_{\mathrm{p}} \sim K_{\mathrm{t}} \sim t^{-1 /\left(C_{\mathrm{p} 2}-1\right)}$.

In this case, both $\epsilon_{\mathrm{p}}$ and $\epsilon_{\mathrm{t}}$ are nonzero; consequently, relaxation to a single-point model will require that $\epsilon_{\mathrm{p}}=\epsilon_{\mathrm{t}}$. But the $K_{\mathrm{t}}$ evolution equation in Eq. (26) implies that necessarily $\epsilon_{\mathrm{p}} \neq \epsilon_{\mathrm{t}}$. Consequently, in the HLS model, decaying turbulence never exhibits spectral equilibrium. This conclusion is not obviously incorrect, but such departures from equilbrium do not appear to have been observed.

Whether or not the constraint $C_{\mathrm{p} 2}<C_{\mathrm{t} 2}$ of Eq. (30) is satisfied by the model Eq. (32) depends on the ratio $K_{\mathrm{p}} / K_{\mathrm{t}}$ during decay. In fact, the values chosen do permit the second solution

$K_{\mathrm{p}}: K_{\mathrm{t}}=0: 1$

with

$K_{\mathrm{t}} \sim t^{-1 /\left(C_{\mathrm{t} 2}-1\right)}$

which, for the constants of Eq. (32) corresponds to

$K_{\mathrm{t}} \sim t^{-7.7}$.

Although the very rapid decay of this solution suggests that it would not be observed in practice, we nevertheless believe that this solution, which corresponds to relaxation to a single-scale model, is more plausible. In conclusion, our purpose is not to uncover flaws in the HLS model, but to draw attention to some physical constraints which appear to be unique to multiplescale models.

\section{Additional realizability constraints on the model coefficients}

The issue of multiple scaling laws does not arise for the selfsimilar growth of RTI-driven turbulence since dimensional considerations force

$$
\begin{aligned}
K_{\mathrm{p}} & =a_{\mathrm{p}} t^{2} \\
K_{\mathrm{t}} & =a_{\mathrm{t}} t^{2} \\
\epsilon_{\mathrm{p}} & =e_{\mathrm{p}} t^{1} \\
\epsilon_{\mathrm{t}} & =e_{\mathrm{t}} t^{1}
\end{aligned}
$$

instead of Eq. (27). In this problem, we find that certain constraints must be satisfied in order that the constants $a_{\mathrm{p}}, a_{\mathrm{t}}, e_{\mathrm{p}}$, and $e_{\mathrm{t}}$ are all positive.

We will analyze this realizability constraint in a more general setting. The generic form of both the $\mathrm{p}$ and $\mathrm{t}$ model equations for the homogeneous case is

$$
\begin{aligned}
\dot{K} & =P-\epsilon, \\
\dot{\epsilon} & =\left(C_{1} P-C_{2} \epsilon\right) \epsilon / K .
\end{aligned}
$$

Here the source term $P \geq 0$ represents some combination of $P_{\mathrm{RT}}$ and $P_{\mathrm{RM}}$ for the $\mathrm{p}$ equations, and it represents $\epsilon_{\mathrm{p}}$ for the t equations. If $P$ is constant, then Eq. (40) is a simple relaxation equation, for which $\epsilon \rightarrow C_{1} P / C_{2}$ on a time scale of $K / \epsilon$. An unphysical, overly damped solution results if $C_{1}>C_{2}$, because $\epsilon$ relaxes to a value greater than $P, \dot{K}$ becomes negative for all remaining time, and $K$ eventually becomes negative.

The same relaxation argument can also be applied to the more complicated form of $P=q K^{a} \epsilon^{b}$, where $q$ is a positive constant. As shown in Eq. (25), this is the form of $P_{\mathrm{RT}}$, with $a=$ $b=1 / 3$, when there is significant scale separation between $k_{0}$ and $k_{1}$ (see Sect. 4). Equations (39) and (40) can then be recast in a similar form for $x \equiv K^{1-a} / \epsilon^{b}$ and $y \equiv \epsilon^{1-b} / K^{a}$ as

$\dot{x}=\left(1-a-b C_{1}\right) q-\left(1-a-b C_{2}\right) y$,

$\dot{y}=\left\{\left[(1-b) C_{1}-a\right] q-\left[(1-b) C_{2}-a\right] y\right\} y / x$.

In this case, the solution relaxes to

$$
\begin{aligned}
& y \rightarrow\left[(1-b) C_{1}-a\right] q /\left[(1-b) C_{2}-a\right], \\
& \dot{x} \rightarrow\left(C_{2}-C_{1}\right)(1-a-b) /\left[(1-b) C_{2}-a\right] .
\end{aligned}
$$

For cases with $1-a-b>0$, the constraint that $x>0$ and $y>0$ for all time requires either

$C_{2} \geq C_{1} \geq a /(1-b)=1 / 2$

or

$C_{2} \leq C_{1} \leq a /(1-b)=1 / 2$.

For the special case $C_{1}=C_{2}=a /(1-b), y$ is constant for all time and one also requires that $q \geq y$, or equivalently that $P \geq \epsilon$ initially.

We stress that these constraints are to be applied to the values of the constants taken in the self-similar state: if $C_{\mathrm{p} 2}$ depends on the turbulence descriptors, as in Eqs. (12) and (32), then $C_{\mathrm{p} 2}$ is found from the solution of a nonlinear equation. Of course, the solution of this equation must be unique; no general criterion can be given to insure uniqueness, which must be checked in each individual case. We remark also that it is not difficult to check that under these constraints, there is only a one-parameter family of similarity solutions of the form Eq. (38) and that all of the constants are determined uniquely in terms of $e_{\mathrm{t}}$.

To use the same set of coefficients for the impulsive RMI case, one must also consider the constraints imposed by the purely decaying case with $P=0$. The exact solution of Eqs. (39) and (40) is then

$\left(K / K_{0}\right)^{C_{2}}=\epsilon / \epsilon_{0}=\left[\left(C_{2}-1\right) t \epsilon_{0} / K_{0}+1\right]^{-C_{2} /\left(1-C_{2}\right)}$ 


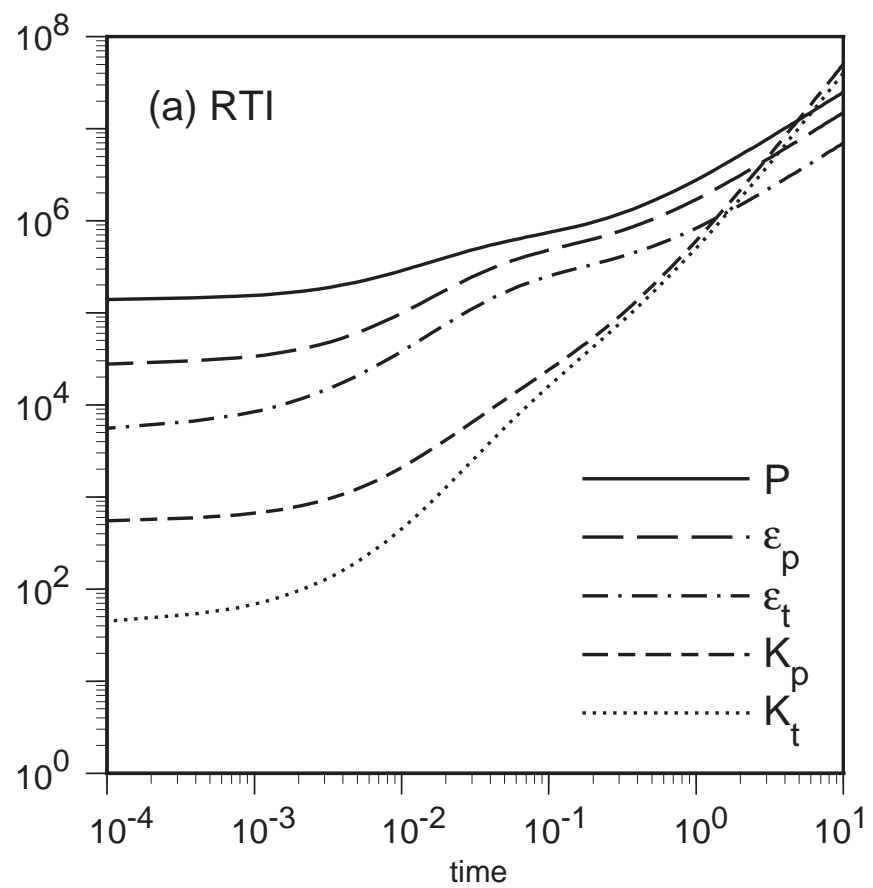

Fig. 4. a) Homogeneous RTI growth using $C_{\mathrm{p} 1}=1.1, C_{\mathrm{p} 2}=1.5$, $C_{\mathrm{t} 1}=1.2, C_{\mathrm{t} 2}=2.0$.

for $C_{2} \neq 1$, and

$K / K_{0}=\epsilon / \epsilon_{0}=\exp \left(-t \epsilon_{0} / K_{0}\right)$

for $C_{2}=1$. Only $C_{2} \geq 1$ leads to physical solutions in this case, so constraint (46) can be excluded. The range of $\mathrm{p}$ model coefficients that give physical solutions for both the RTI and RMI cases is therefore given by $C_{\mathrm{p} 2} \geq 1, C_{\mathrm{p} 2} \geq C_{\mathrm{p} 1}, C_{\mathrm{p} 1} \geq 1 / 2$. The RMI case also requires $C_{\mathrm{t} 2} \geq 1$ for the $\mathrm{t}$ model equations. The $\mathrm{t}$ model coefficients are not as well constrained in the RTI case, where the source term $P=\epsilon_{\mathrm{p}}$. Because the $\mathrm{p}$ equations in Eq. (25) are decoupled from the $t$ equations in the absence of diffusion terms, $\epsilon_{\mathrm{p}}$ must be considered to be a general time-dependent forcing term. However, constraint (30), derived in the previous section to give a single-scale model at late times, requires $C_{\mathrm{t} 2}>C_{\mathrm{t} 1}$, which generally suffices to give physical solutions. Figure 4a shows RTI growth for the homogeneous model equations with $C_{\mathrm{p} 1}=1.1, C_{\mathrm{p} 2}=1.5, C_{\mathrm{t} 1}=1.2$, and $C_{\mathrm{t} 2}=2.0$; at late times $P, \epsilon_{\mathrm{p}}, \epsilon_{\mathrm{t}} \propto t$ and $K_{\mathrm{p}}, K_{\mathrm{t}} \propto t^{2}$. The same model coefficients are used for RMI decay following an initial production pulse, shown in Fig. $4 b$, which exhibits the same late-time behaviour as shown in Fig. 1 for the case $C_{\mathrm{t} 2}>C_{\mathrm{p} 2}>C_{\mathrm{t} 1}$.

To illustrate the transient dynamics, two further cases of RTI growth are presented in Figs. 5 and 6 . In the first, the initial conditions satisfy $K_{\mathrm{p}}=10 K_{\mathrm{t}}$, and in the second, $K_{\mathrm{t}}=10 K_{\mathrm{p}}$. These somewhat artificial initial conditions were chosen to illustrate how the model can generate either $K_{\mathrm{p}}$ or $K_{\mathrm{t}}$ from states in which they are initially small.

The delayed growth of the small scale turbulence which is evident in Fig. 5 and even more prominent in Fig. 6, might be relevant to numerical simulations of type II supernova explosions (Müller 1991; Kifonidis et al. 2000). Such simulations

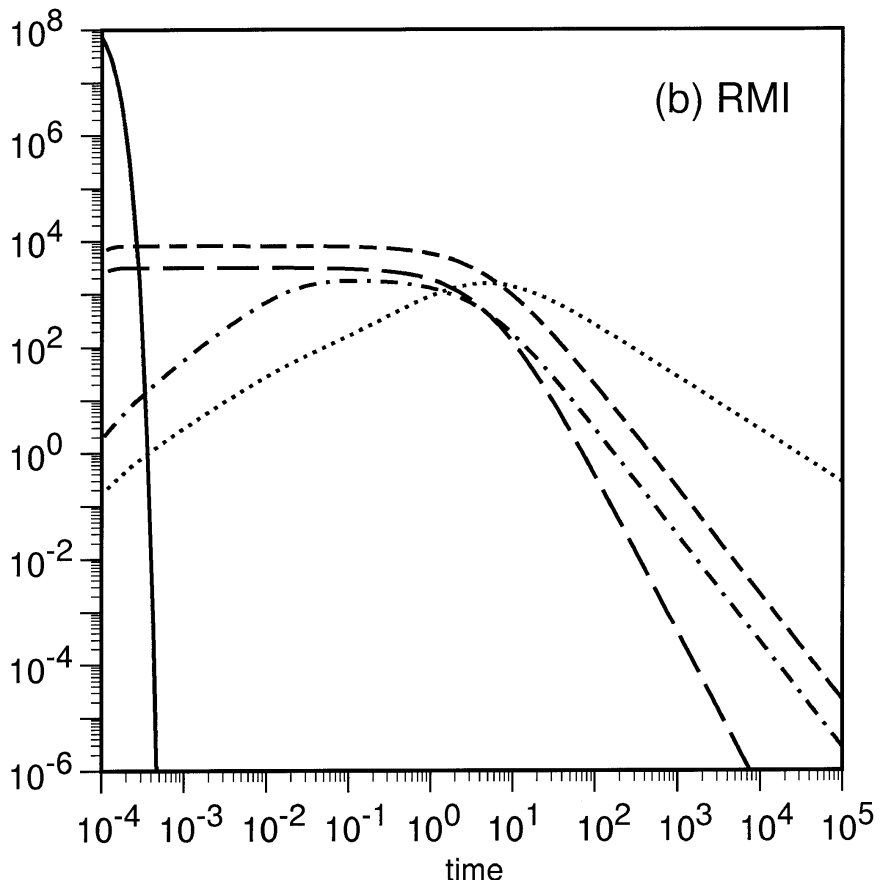

Fig. 4. b) Homogeneous RMI decay, using the same coefficient values as in a); curve labels are as in Fig. 4a.

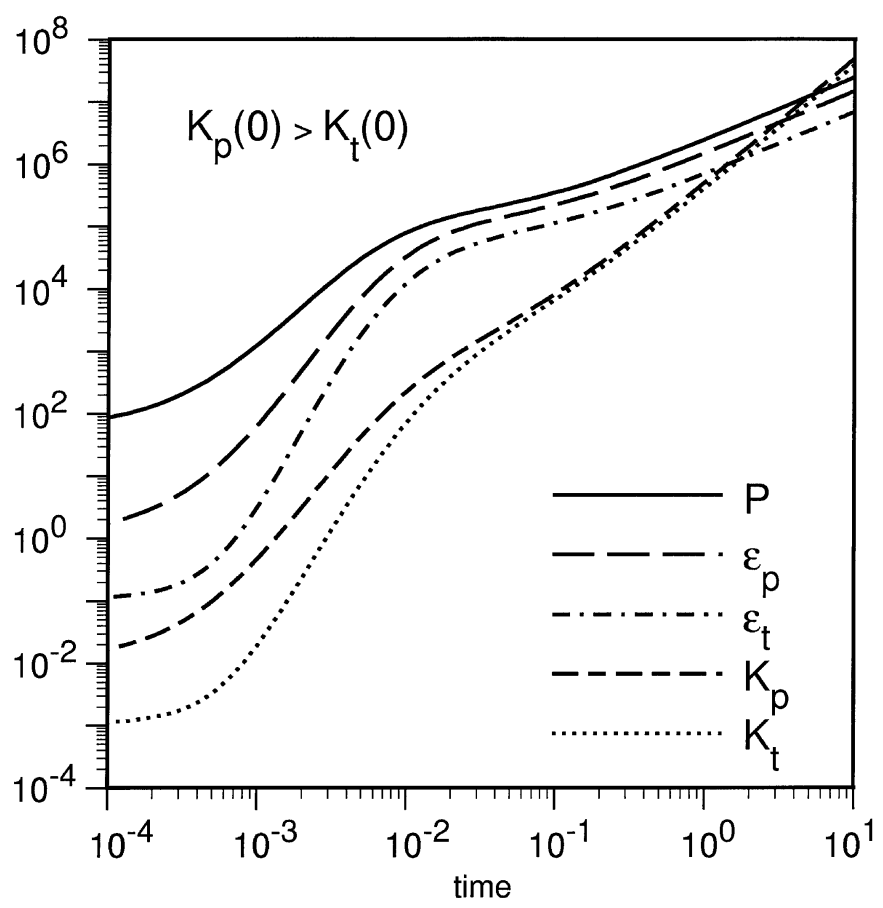

Fig. 5. RTI growth beginning from a state with $K_{\mathrm{p}}=10 K_{\mathrm{t}}$.

consistently underpredict the penetration of heavy elements like $\mathrm{Ni}$ into the photosphere.

A possible connection is the following: numerical methods inevitably generate artificial viscosity; if this viscosity acts to overpredict mixing at small scales during early phases of the evolution, delayed release of heavy elements might be predicted, even though the numerical methods attempt direct simulation and do not explicitly consider turbulence. If so, this would be an argument for the use of the two-scale model as 


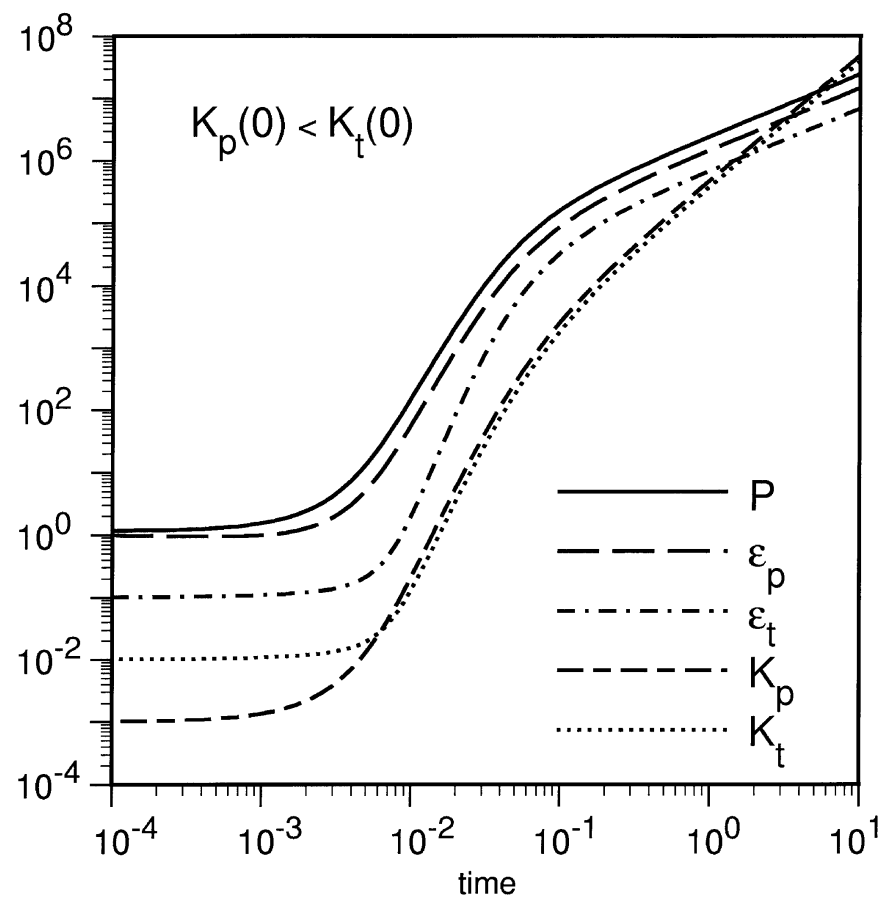

Fig. 6. RTI growth beginning from a state with $K_{\mathrm{t}}=10 K_{\mathrm{p}}$.

a subgrid model in large-scale numerical simulations. At this point, however, this connection can only be suggested speculatively.

\section{Summary and conclusions}

1. The turbulent flows induced by RTI and RMI are particularly appropriate for a two-scale modeling approach. We developed such model in which energy transfer through large scales can be distinguished from viscous dissipation.

2. Multiple-scale models must permit relaxation to a unique self-similar evolution. Conditions which insure this relaxation are derived in the special cases of decaying turbulence, appropriate to RMI-induced turbulence following the passage of the shock, and of growing RTI-induced turbulence.

Acknowledgements. The authors are grateful to the anonymous referee and to Dr. Dmitri Ryutov of Lawrence Livermore National Laboratory for suggesting the possible relevance of this work to supernova explosions.
This work was performed under the auspices of the U.S Department of Energy by the University of California Lawrence Livermore National Laboratory under contract No. W-7405-Eng-48.

\section{References}

Arnett, W. D., Bahcall, J. N., Kirshner, R. T., \& Woosley, S. E. 1989, ARA\&A, 27, 629

Bell, T. L., \& Nelkin, M. 1978, J. Fluid Mech., 88, 369

Besnard, D. F., Harlow, F. H., Rauenzahn, R. M., \& Zemach, C. 1996, Theor. Comput. Fluid Dyn., 8, 1

Cadiou, A., \& Hanjalić, K. 1999, One-point multi-scale turbulence closure derived from a spectral description, TU Delft Report APTF R/99-11

Chandrasekhar, S. 1961, Hydrodynamic and Hydromagnetic Stability (Oxford: Oxford Univ. Press)

Haan, S. W. 1991, Phys. Fluids B, 3, 2349

Hanjalić, K., Launder, B. E., \& Schiestel, R. 1980, in Turbulent Shear Flows II (London: Springer)

Kifonidis, K., Plewa, T., Janka, H.-Th., \& Müller, E. 2000, ApJ, 531, L123

Kolmogorov, A. N. 1941, Dokl. Akad. Nauk SSSR, 30, 301

Kraichnan, R. H. 1959, J. Fluid Mech., 5, 497

Kraichnan, R. H. 1971 J. Fluid Mech., 47, 513

Laporta, A. 1995, Thesis, École Centrale de Lyon

Ledoux, P., Schwarzschild, M., \& Spiegel, E. A. 1961, ApJ, 133, 184

Lindl, J. 1997, Internal Confinement Fusion: The quest for ignition and energy gain (New York: Springer)

Matthaeus, W. H., \& Zhou, Y. 1989, Phys. Fluids B, 1, 1929

Meshkov, E. E. 1969, Izv. Acad. Sci. USSR Fluid Dyn., 4, 101

Mikaelian, K. O. 1989, Physica D, 36, 343

Müller, E., Fryxell, B., \& Arnett, D. 1991, A\&A, 251, 505

Lord Rayleigh 1883, Proc. Roy. Math. Soc., 14, 170

Remington, B. A., Drake, R. P., Takabe, H., \& Arbett, D. 2000, Phys. Plasmas, 7, 1641

Richtmyer, R. D. 1960, Commun. Pure Appl. Math., 13, 297

Ryutov, D., Drake, R. P., Kane, J., et al. 1999, ApJ, 518, 821

Schiestel, R. 1987, Phys. Fluids, 30, 722

Smarr, L., Wilson, J. R., Barton, R. P., \& Bowers, R. L. 1981, ApJ, 246, 515

Taylor, G. I. 1950, Proc. Roy. Soc. London, Ser. A, 201, 192

Zhou, Ye 1993a Phys. Fluids A, 5, 1092

Zhou, Ye 1993b Phys. Fluids A, 5, 2511

Zhou, Ye 1995, Phys. Fluids, 7, 2092

Zhou, Ye, Yeung, P. K., \& Brasseur, J. G. 1996, Phys. Rev. E, 53, 1261

Zhou, Ye 2001, Phys. Fluids, 13, 538 